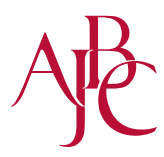

\title{
The Holistic View of Cosmetic Science
}

Yinmao Dong, Xianrong Qiu, Hong Meng*

Department of Cosmetic Science and Technology, Beijing Technology and Business University, Beijing, China

\begin{abstract}
*Corresponding author: Hong Meng, Department of Cosmetic Science and Technology, College of Science, Beijing Technology and Business University, No. 11/33, Fucheng Road, Haidian District, Beijing, China Tel.: +861068987110 Email: menghong2000@163.com
\end{abstract}

Received February 22, 2016

Revised March 22, 2016

Accepted March 23, 2016

Published March 30, 2016

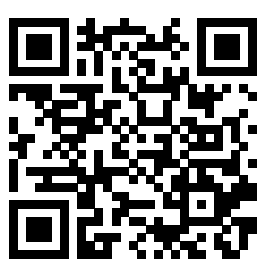

\begin{abstract}
Purpose: Cosmetics science has become an independent and comprehensive science with over 20 disciplines, including life science, chemistry, packaging design. In the cosmetic skin science, there were early simple skin histology, and were the understanding of the hypothalamus pituitary adrenal axis in skin, that the skin becomes the target organ of various endocrine signaling. There were early the macro understanding of the skin, and now the micro understanding of the skin, such as skin microbial community and so on. Therefore, this paper put forward a holistic view to solve skin problems. Methods: Inspired from the formation and significance of holism at all times and in all countries, we expounded the development history of cosmetic science and cosmetics related disciplines, especially dermatology. Results: This summarized the development of dermatology and established a holistic view to remove various skin problems. Conclusion: The holistic view of cosmetic science will become an inevitable trend in the development of cosmetic science.
\end{abstract}

Keywords: Holism, Cosmetic science, Dermatology, Skin symptom, Cosmetics formula design

\section{Introduction}

讨论 “化妆品科学的整体观” ，首先要知道整体观的概 念, 然后再讨论整体观可能对化妆品科学的发展的影响。通 过万方数据知识服务平台以 “整体观” 为主体词查询文章, 没有发现准确表述整体观的文章。在百度上搜索“整体观” 词条, 百度百科显示: 整体观是指从全局考虑问题的观念。 整体观, 首先是指自然界本身是一个整体, 人和其他的生 命、生物都是其中的一部分。如果这个整体或某一部分受到 损害, 那么其他方面也将受到影响, 整体则因之破坏。不论 概念准确与否, 基本上描绘出了中心意思。

有史以来, 人类的哲学思想在公元前 800 年至 200 年得到实 质性的发展, 史上称之为 “轴心时代”。在中国的古代, 系 统整体观已开始萌芽、生长。《周易》将天地视为自然与人 类的创生者, 所谓 “有天地然后万物生焉”, “有天地然 后有万物”（Qi \& Zhu, 2013）。儒家 “天人合一”三才论 的自然整体观从《周易》发轫, 经过孔子、孟子、荀子、 董仲舒的确立和发展, 到宋明理学时代趋于成熟 (Ren \& Li, 2006）。老子的整体观思维模式, “道生一, 一生二, 二生
三，三生万物”；“人法地，地法天，天法道，道法自然”

（Tang, 2012）。墨子的 “一”与 “多” , 惠施的 “至大无 外，至小无内” 等论述，都在一定程度上揭示了客观事物的 整体性及辩证的层次关系。《黄帝内经》中的整体性观点最 丰富, 认为人体器官的功能既有区别, 又有联系, 构成一个 有机整体。在整体观的指导下, 认为人体某部分发生病变, 可影响到整个身体或器官, 全身的状况又可影响到局部的病 理变化。《黄帝内经》还论述了人体与外界环境的关系, 如 将人体的某些基本问题与四时季节变化、地理水土、社会生 活、思想情绪等方面的变化进行联系, 形成了人体与外界环 境相互感应的观点（Xu \& Wang, 2014）。在古代西方, 系统 整体观同样孕育、生长, 例如古希腊哲学家赫拉克利特在 《论自然界》中把世界看作 “包括一切的整体”。爱利亚学 派的创始人巴门尼德认为 “存在” 就是整体, “存在不生不 灭, 它是整体, 唯一和不动”。因为它的各个部分都完全相 同, 并且紧密联系在一起, 因此它是不可分割的, 是一个整 体。恩培多格勒认为, 世界万物的本原是火、水、土、气 四种元素, 即“四根”。世界上千差万别的事物都是由 “四 
根” 组成的。然而, 任何一个整体物并不是各种元素即部分 的简单组合和相加, 整体具有部分所不具有的特性 (Mao, 2006)。

这种早期的整体观认识, 中国与西方具有惊人的相似, 这 种哲学思想的发生、发展以及内容相似程度让人难以相信。 但是，随着历史发展，不同区域人们与自然的关系不同，生 活方式在发展方向上, 具有巨大的差异。中国属于东方文 明，一直以来为农耕经济，中央集权的管理方式，形成大河 文明; 在遥远的古老西方，较多从事海外贸易经济，民族法 治制度的管理模式，形成海洋文明。中国对古老的认识世界 的系统整体观方法并没有发生变化，经过一代又一代的先哲 们不断整理、完善和丰富; 然而, 在西方尤其是亚里士多德 比较详细地论述了系统整体观，概括起来有两种含义，一是 从逻辑的定义出发, 部分是包括在整体之中的。如一个音节 由几个字母组成, 这些字母就是音节的部分, 音节包括字 母; 二是对有机的统一体来说，其部分离开整体就不成为部 分, 只有在与其他部分处于有机联系之中, 它才成为有机整 体的一部分（Tang, 2002）。然而, 他的整体观的第一层意 思在西方后来发展过程中起着主导作用, 逐渐形成现代的科 学思维（数学的、逻辑的思维方式）。随着西方列强文化入 侵以及武力入侵中国, 中国的传统整体观受到西方的现代科 学思维巨大冲击, 甚至出现中国学者公开否定中国传统文化 以及认识自然的能力。

在相当长的历史时期, 这种科学的逻辑思维, 促进着人类 的发展。1951年萠因在《经验论的两个教条》中正式提出还 原论(reductionism)一词（Huang, 2008）。从科学和哲学思 想上说, 还原论和分析思维的主要奠基者是笛卡尔。广义还 原论的最基本内涵, 自然界中所有的现象都能够被还原为某 种自然的基本规律，它的总特征是自然的复杂性。基于这种 认识, 笛卡尔提出适用于指导人们思维活动的四条原则, 相 当完整地规定了还原论和分析思维的基本内涵：第一，凡是 没有明确地认识的东西, 决不把它当成真的接受; 第二, 把 所审查的每一个难题按照可能和必要的程度分成若干部分, 以便一一解决; 第三, 按次序进行思考, 从最简单、最容易 认识的对象开始, 一点一点逐步上升, 直到认识最复杂的对 象。就连那些本来没有先后关系的东西, 也给它们设定一个 次序; 第四, 在任何情况之下, 都要尽量全面地考察, 尽量 普遍地复查, 做到确信毫无遗漏。

随着人类认识世界和利用世界的能力逐渐增强, 从整体, 到部分、到基本单位，到分子、核子、原子、中子、量子 等逐步深入, 已经有些现象无法用更小的部分进行解释, 西方科学精英们又开始讨论整体观。1926年, 斯穆茨创用 “Holism”一词，即“整体观”，用以指“宇宙中制造或创
生整体的根本作用要素”。维基词典中将Holism解释为某种 体系 (如物理、生物、化学、社会、经济、精神, 以及逻辑 等）和及其属性应当被看着一个整体，而不是简单的组合。 体系的功能被视为一个整体, 单独的某一组成部分是不能够 体现整体功能。使用Holism一词, 在Pubmed上进行搜索, 从1970s初, 科学家们便在生命科学界开始大讨论 (Torday, 2015; Ellerby et al., 2000; Kunitz, 2002; Röhl, 2012）。

学者们普遍认为还原论的分析观是一种只见树木不见森林 的片面观点。它在科学发展的一定时期对于科学的进步来说 是完全必要的, 甚至是必不可少的。但当科学的知识积累到 一定程度, 当人们的认识由一个个点发展到需要进一步弄清 这些点之间的联系, 需要把个别的知识综合起来, 还原论纲 领的局限性就越来越凸显。复杂性思维模式的形成, 就代表 了科学研究模式的一种转变, 超越还原论也就成为一种必然 趋势, 使用整体观将逻辑科学思维的产物整合起来, 将促进 人类文明的发展。

为此, 在阐述的上述整体观与还原论后, 谈论化妆品科学 的整体观, 就比较容易。下文将从化妆品的发生发展, 以最 早人类装扮目的, 发展到现在护肤目的; 从对皮肤简单的组 织、生理、生化认识, 到目前了解和熟知皮肤功能调节, 即 “下丘脑-神经-免疫”功能轴的调节方式，陈述化妆品科学 的整体观。为此, 化妆品科学的研究内容和对象不能再是简 单 “头痛医头脚痛医脚” , 对研究和处理任何一个皮肤问题 必须建立整体观。

\section{Cosmetics and cosmetic science}

\section{The development of cosmetics}

在古代中国，妆也作 “装”、“粧”、“䊫”。意思是 “打扮”、“修饰”。唐朝白居易《琵琶行》“曲罢曾教善 才伏, 妆成每被秋娘妒”。也表示妆饰用物和服装。乐府诗 集二五木兰诗 “阿姊闻妹来, 当户理红妆” 等。化妆, 涂脂 抹粉以化妆。中国传统戏曲按照不同角色行当化妆, 丑行 和净行一般使用脸谱。在西方, 化妆品一词来源于希腊语 koountIK TXVn (kosmetikē tekhnē), 意义是 “着装和修饰技 术”。

原始的化妆品, 是使用天然的动植物油脂对皮肤作单纯的 防护, 即直接使用动植物或矿物来源的、不经过化学处理的 各类油脂。如古埃及人四千多年前就已在宗教仪式上以及皇 朝贵族个人的护肤和美容上, 使用了动植物油脂、矿物油和 植物花朵。古罗马人对皮肤、毛发、指甲、口唇的美化。与 此同时, 中国化妆品也已有了长足的发展, 在古籍《汉书》 
中就有画眉、点唇的记载, 《齐民要术》中介绍了有丁香芬 芳的香粉，中国宋朝韩彦直所著《枯隶》是世界上有关芳香 方面较早的专门著作。

十八、十九世纪欧洲工业革命后, 化学、物理学、生物学 和医药学得到了空前的发展, 许多新的原料、设备和技术被 应用于化妆品生产, 更由于以后的表面化学, 胶体化学、结 晶化学、流变学和乳化理论等原理的发展, 解决了正确选择 乳化剂的关键问题, 逐渐发展成为一门新的专业性的科学技 术。

植物化学分析和提取技术的革新, 使某些动植物萃取精华 添加到化妆品中成为可能，如从皇角、木瓜等天然植物提取 的精华，或者从动物皮肉和内脏中提取的蛋白和激素样物质 等精华素, 从此便赋予了化妆品具有某些功能性如美白、祛 粉刺、祛斑、抗皱等成为可能。

科学技术的不断进步，对皮肤生理病理有着深入了解，对 化妆品的发展起到了很大促进作用。采用生物技术制造与人 体自身结构相仿, 制造出具有高亲和力的生物精华物质, 并 复配到化妆品中, 以补充、修复和调整细胞来达到抗衰老、 修复受损皮肤等功效，如生物工程制剂神经酰胺、表皮生长 因子等（Liuetal.,2014）。

\section{Cosmetics: an independent subject}

中国化妆品定义：以涂搽喷酒或者其他类似的方法，散布 于人体表面任何部位(皮肤、毛发、指甲、口唇等)，以达到 清洁、消除不良气味、护肤、美容和修饰目的的日用化学工 业品。我国新的法规将牙膏、漱口水等口腔清洁和保健用品 也划入了化妆品的范畴。

从上述定义可以看出，化妆品并非单纯的装饰或修饰，并 已经含盖清洁、消除不良气味和护肤, 化妆品逐渐发展成为 一门独立的学科。化妆品学, 是集生命科学、皮肤科学, 化 妆品原料科学、配方科学, 以及制造工艺、生产管理科学, 以及有关法规和消费者管理等一系列学科的一门综合性学 科。它又是集化学、医学、药学、皮肤科学、齿学、生物化 学、物理化学、化学工艺学、流变学、美学、色彩学、生理 学、心理学、管理学和法律学等相关科学于一身的应用学科 (Liangetal.,2015)。

\section{The development of dermatology}

皮肤具有不同的颜色，表面拥有不同种类的细纹或皱褶， 可以看到汗毛、胡须、眉毛、头发等, 由于不同人、不同风 俗、不同民族、不同国度对上述皮肤特点喜好不同，逐渐形
成了不同的审美观, 为此皮肤也成为审美的重要器官; 由于 皮肤是机体的一部分, 参与机体对外界物质的吸收、分布、 代谢和排泄、调节体温, 肩负着感知外界、皮肤免疫等重要 的生理功能，为机体健康状况的晴雨表。

\section{Skin histology}

由浅入深，皮肤由表皮、真皮及皮下组织组成，及多种附 属器官如皮脂腺、汗腺、毛囊, 分布着丰富的细胞、血管、 淋巴、神经等。

表皮层, 主要是角质形成细胞, 从表面到基底共分为 5 层, 依次为角质层、透明层、颗粒层、棘层、基底层。在表皮角 质形成细胞之间, 有序地分布中一些树突状细胞, 包括黑素 细胞、Langerhans细胞和Merkel细胞, 这些细胞与皮肤和机 体免疫、炎症反应等相关。黑素细胞不但参与上述反应, 还 是决定肤色的主要因素。上述细胞之间除具有丰富的细胞间 质以外，细胞与细胞之间还具有物质交换、信息传递等功 能。

真皮层, 主要细胞成分为成纤维细胞, 细胞呈梭形或扁的 星状, 具有突起。根据不同的功能活动状态, 将细胞分为成 纤维细胞和纤维细胞。成纤维细胞乃是功能活动旺盛的细 胞, 具明显的蛋白质合成和分泌活动; 纤维细胞功能活动不 活跃, 此二型细胞可互相转化。细胞外基质由细胞分泌到 胞外间质中的大分子物质组成，可影响细胞分化、增殖、黏 附、形态发生和表型表达等生物学过程。成分包括：胶原蛋 白、弹性蛋白、蛋白多糖和糖蛋白等。

皮下组织位于真皮下方，与真皮无明显的界限，由疏松结 缔组织和脂肪小叶构成, 又称为皮下脂肪层。此层还含有汗 腺、血管、淋巴管和神经等。皮下组织是热的绝缘体及储藏 热能的仓库, 尚可缓冲外来的冲击, 适度的皮下脂肪可使人 显得丰满, 皮肤细淢、柔嫩、红润光泽、富有弹性, 显示出 人体美的质感与动感。

为此, 对机体而言, 皮肤不仅是保护机体避免水分蒸发、 营养物质流失等, 完美天然物理屏障即机械性屏障; 还是一 个完整的、具有继消化道、呼吸道之后的第三大吸收器官, 继消化道、呼吸道和泌尿系统之后的排泄器官; 负责体温 调节、感知外界环境变化的重要器官; 肩负着机体的部分免 疫、内分泌等功能。

\section{Functional regulation of skin}

皮肤是神经、体液内分泌的靶器官。皮肤作为神经、体液 内分泌的靶器官之一, 在接受神经递质调节的同时, 也受到 体液内分泌的调节作用（Liao et al., 2012）。 


\section{1) Neuroregulation}

皮肤是机体表面的一种保护性感觉器官, 神经末梢十分丰 富, 这些神经末梢将感觉到的外围环境中各种刺激（物理 性、化学性或生物性) 信号, 通过神经纤维反应到大脑, 大 脑经过处理再反映到皮肤。上述神经纤维为感觉神经纤维, 神经末梢表现为Merkel细胞轴索复合体。除感觉神经纤维之 外, 还存在植物神经纤维, 没有神经髓鞘, 分支直接渗透 到真皮。这些植物神经来源于交感神经系统, 与血管、立毛 肌、大小汗腺功能相关。

皮肤与神经具有发育上的同源性, 均由细胞的外胚层分化 而来。人的精神状态、心理变化经过神经传递, 极大地影响 着皮肤。当精神受到创伤、心情忧郁、压力过大并长期受到 刺激时, 会使交感神经失去平衡, 影响皮肤的营养, 出现干 燥、松弛、无光泽、过早衰老等现象, 同时还会降低皮肤的 免疫力而易发生一些损容性皮肤病。其实, 消极心理因素对 人体健康危害极大, 当人体处于长期不良且强烈的 “负性情 绪” 状态, 必然直接导致心理活动失衡, 影响身体健康, 而 皮肤往往是不良情绪的 “靶器官” , 自然会严重地危害到皮 肤的健康而出现面色苍白、晦暗无光泽, 皮肤粗䊁、松弛无 弹性, 色素沉着, 面部皱纹增多、加深等症状（Liao et al., 2012）。

\section{2) Humoral and endocrine regulation}

皮肤是下丘脑-垂体一肾上腺轴的内分泌靶器官, 即多种内分 泌信号作用的靶器官。当精神受到创伤、心情忧郁、压力过 大并长期受到刺激时, 可以导致激素失调, 内分泌紊乱。皮 肤作为激素作用的终末器官, 当遭受损伤, 进行修复和再生 时必定受其影响（Liu\&Lei, 2009; Guo \& Hao, 2009）。性激素 影响正常皮肤的真、表皮厚度, 有丝分裂能力和血管化水 平, 以及弹性蛋白的特征和胶原组织的含量, 影响着机体皮 肤表面特征和组织学特征, 在实际生活中, 可以看到不同年 龄、不同性别皮肤表面特征表现出比较大的差异。雌激素通 过对炎症反应、基质沉积、表皮化和瘢痕成熟等环节影响皮 肤愈合与再生, 局部应用雌激素不仅加强女性也加强男性患 者创面的愈合能力。在皮肤创面, 雌激素可以刺激巨嗜细胞 产生神经生长因子(NGF)以及血管内皮细胞生长因子(VEGF), 刺激角质形成细胞产生粒细胞-巨细胞集落刺激因子(GMCSF), 刺激纤维原细胞产生成纤维细胞生长因子(FGF)和转化 生长因子(TGF), 这些因子增加了创面神经和表皮的再生以及 肉芽组织的形成。在创面愈合过程中, 皮肤的炎症细胞、角 质形成细胞及纤维原细胞中均有雄激素受体(AR)的表达, 雄 激素延长炎症反应, 减少创口细胞外基质沉积, 使伤口愈合 速度减慢。甲状腺激素通过角蛋白基因的表达对创面愈合和 再生也有很大的影响, 局部运用甲状腺素治疗将加速创口的
愈合（Liao et al., 2012）。

\section{3) Skin auto control system}

皮肤受机体神经内分泌调节的同时，也存在自分泌和旁分 泌调节。大量的研究证据表明, 皮肤及其附属器作为外周的 神经器官, 它一方面除了受到传统中枢性的应激调节外, 另 一方面也可以起到独立的神经调控作用。在皮肤组织中存在 着大量的神经, 这些神经组织在皮肤的表皮与真皮间相互渗 透穿行, 形成三维立体网络。另外, 皮肤之所以作为神经调 节的靶器官之一, 还因为在皮肤组织中存在大量具有激素或 神经递质的受体, 并自身能够表达各种类型激素或神经递质 的细胞, 如: 角质形成细胞、黑素细胞、Merkel细胞、朗格 汉斯细胞、肥大细胞、成纤维细胞、脂肪细胞、汗腺细胞、 皮脂腺细胞等。这些细胞通过神经的信号介导, 有效调节皮 肤功能的完整。

\section{(1) Keratinocyte}

角质形成细胞，在皮肤的最表层（Gandarillas, 2012）。组 织学显示角质形成细胞之间混杂着其它细胞, 尤其是基底部 的黑素细胞, 角质形成细胞与黑素细胞树突接触并接受黑素 小体转输。随着科学技术进步, 发现角质形成细胞与黑素细 胞之间, 以及其它皮肤细胞之间, 并非简单的细胞与细胞之 间接触这么简单。例如, 单独培养的角质形成细胞经过紫外 线照射后，其培养液能够促进黑素细胞增殖，并发现，不同 时期的角质形成细胞对黑素细胞的调控作用不一样, 未分化 的角质形成细胞对黑素细胞的生长、形态以及表面抗原的表 达起调控作用; 而分化的角质形成细胞对黑素细胞的调控 作用甚微（Liu et al., 2014）。研究发现, 在紫外线照射、皮 肤炎症、外伤等因素作用下, 角质形成细胞分泌多种细胞因 子, 主要有碱性成纤维细胞因子(bFGF)、内皮素-1(ET-1)、神 经生长因子(NGF)、白介素-1 $\alpha($ (IL-1a) 、白介素-6(IL-6)、肿瘤坏 死因子- $a($ TNF- $a$ )、干细胞因子（SCF）等（Liao et al., 2012; Manetal.,2008) 。

\section{(2) Melanocyte}

黑素细胞产生的黑素是决定皮肤颜色的主要因素。黑素细 胞合成黑素主要发生在黑素小体, 以酪氨酸为底物, 在酪氨 酸酶的作用下合成黑素。黑素细胞酪氨酸酶的活性除了取决 于自身因素外, 还要受到生长环境中细胞因子的调节。表皮 中许多细胞因子都可以由角质形成细胞产生, 如: b-FGF、 ET-1、NGF、IL-1、IL-6、TNF- $a 、$ SCF等。大量研究表明, 这 些因子对黑素细胞的增殖、形态及黑素合成有着或正或负的 影响, 提示表皮内角质形成细胞可以通过产生各种细胞因子 
调节黑素细胞的生物学性（Liao et al., 2012; Wen et al., 2014; Cichoreket al.,2013)。

\section{(3) Fibroblast}

在组织学或功能学上看, 成纤维细胞似乎只是环境因素改 变的情况下, 产生相应的功能调节, 增强或减弱合成胶原纤 维、弹力纤维或其它基质。然而, 研究发现成纤维细胞能够 分泌许多神经递质、激素或细胞因子。如肿瘤坏死因子、P 物质、 $\beta$-内啡肽、促肾上腺皮质激素 (ACTH) 、 $\alpha$-黑素细胞 刺激激素 (a-MSH) 等 (Liu, 2003)。

\section{(4) Adipocyte}

近年来逐渐对皮下组织及皮肤附件特别是脂肪细胞, 间质 细胞认识的深入, 不再简单地将脂肪组织仅仅看成是能量咜 存器官, 而且将其作为性激素的代谢器官以及内分泌器官。 脂肪组织能够产生大量的生物活性肽, 包括脂肪因子, 瘦 素、脂蛋白酶、抵抗素、血管紧张素原等, 在局部与脂肪 细胞表面特异性受体结合, 以自分泌和旁分泌的形式发挥作 用。已经确认, 瘦素参与内分泌、炎症反应、具有促血管和 肉芽组织形成和再上皮化的潜能。脂肪细胞因子作为炎性因 子也参与血管内皮细胞功能调节（Jangetal., 2012）。

\section{(5) Vascular endothelial cell}

血管内皮细胞为心血管内腔面的单层表皮细胞, 它不仅仅 具有选择性的通透、抗血栓、调节血管紧张度、促进毛细血 管形成以及产生血细胞粘接因子等功能, 而且还是多种血管 活性物质、细胞因子以及生长因子的来源和靶器官。

\section{(6) Langerhans cell}

朗格罕细胞能够产生阿片黑素原 (POMC) , 是 $\alpha$-黑素细胞 刺激激素、促肾上腺皮质激素和 $\beta$-吲哚酚的前体。皮下组织 中的其它免疫细胞, 能分泌多种神经递质或内分泌激素, 如 去甲肾上腺素、乙酰胆碱(Ach)、5-羟色胺(5-HT)、生长激素 $(\mathrm{GH})$ 、催乳素(PRL)、甲状腺激素, 促甲状腺素, 促肾上腺皮 质激素、 $\alpha$-黑素细胞刺激激素、P物质，血管活性肠肽、降 钙素基因相关肽(CGRP)、 $\beta$-内啡肽等。另一方面，免疫细胞 上亦存在众多的递质和激素受体。这就为神经及内分泌系统 作用于免疫系统奠定了物质基础。

\section{(7) The emerge of new conceptions}

皮肤组织神经-免疫系统: 往常人们把神经系统、免疫系统 和皮肤器官看成彼此之间各自独立的, 然而这三者在解剖 结构、生理功能、以及产生的某些病理过程等, 是紧密相
连的, 有人便提出 “神经免疫皮肤系统” (NICS) 的概念 （Liu, 2009; Guo \& Hao, 2009）。在神经纤维、免疫细胞和皮 肤细胞三者之间, 存在着细胞与细胞之间物理性接触; 存在 着各自分泌的细胞因子或介质，得到彼此之间受体接受和调 节, 即存在着化学接触; 有着协调的功能调节, 如紧张或焦 虑出现的排汗现象, 可以称得上功能接触; 皮肤遭受外界有 害因素侵害时, 包括物理性、化学性和生物性损害, 均会引 起皮肤细胞分泌介质, 调节免疫, 出现炎性反应等过程, 被 认为是免疫接触。随着科学技术的进步, 普遍认为皮肤的神 经内分泌功能由皮肤的神经内分泌单位完成, 神经内分泌单 位包括角质形成细胞、黑素细胞、成纤维细胞和免疫细胞, 这些细胞通过自分泌及旁分泌方式相互作用, 形成完整有序 的神经内分泌网络（Arcketal.,2006）。

皮肤组织下丘脑-垂体-肾上腺系统：皮肤与中枢神经系统具 有相同的神经外胚层来源, 皮肤密布复杂的神经并表达多种 激素及其受体，暗示着皮肤与神经及内分泌系统的可能联 系。目前认为皮肤是外周的神经内分泌器官, 皮肤具有和 中枢相似的下丘脑-垂体-肾上腺(HPA)系统。皮肤本身的细胞 能够合成和释放激素，如甲状旁腺素相关蛋白、POMC衍生 物、 $a$-黑素细胞刺激激素、肾上腺皮质激素、 $\beta$-内啡肽、促 肾上腺皮质索释放激素、皮质激素肽和神经递质儿茶酚胺、 乙酰胆碱、生物源胺的前体。这些局部产生的激素和神经递 质以旁分泌或自分泌方式起作用。皮脂腺无神经支配, 其功 能调节是旁分泌和自分泌调节的最好例证 (Roosterman et al.,2006)。

\section{Skin micro-ecosystem}

微生态学 (microecology) 全称为微观生态学。微生态学是 研究正常微生物群与其宿主( 人、动物、植物)相互关系的生 命科学分支, 是细胞水平、分子水平的生态学。人体微生物种 类繁多, 数量巨大, 他们共同组成了人体微生态系统。所以 学者按照正常微生物群在微生态系统中所占的空间不同把人 的微生态系统分为以下几类：人类口腔微生态系统、人类胃 肠道微生态系统、人类泌尿道微生态系统、人类生殖道微生 态系统、人类皮肤微生态系统和人类呼吸道微生态系统。这 些微生态系统与人体建立起密切的关系, 对促进人体生理机 能的完善尤其是免疫功能的成熟起非常重要的作用。它们与 机体已形成相互依存、互为利益、相互协调又相互制约的统 一。这种统一体现了人类微生态的动态平衡, 平衡则健康, 失衡则致病（Fang, 2014; Prohicetal., 2014）。 近年来, 微生态与人类健康的关系成为热门课题。随着研 
究方法的进展, 宏基因组学的应用, 微生态与人体健康之间 的关系并得到长足发展。

\section{Relationship between skin Micro-ecosystem and skin health}

(1)皮肤常驻菌具有占位保护作用, 有层次有序地定植在皮 肤上, 犹如一层生物屏障, 使致病菌及外籍菌无法立足于皮 表; (2)多达20\%的常驻菌可产生抑制病原菌的化合物。如 革兰阳性共生菌, 包括乳酸菌、链球菌、葡萄球菌可产生杀 菌素, 表皮葡萄球菌可产生一种抗菌肽酚可溶性调控蛋白, 对金黄色葡萄球菌、A 组链球菌、大肠杆菌具有选择性杀灭 作用，但不针对其他表皮葡萄球菌及常驻菌; (3)常驻菌可分 解皮脂甘油三酯为脂肪酸, 形成乳化皮脂膜, 既对自身及表 皮角质形成细胞具有营养作用, 又可防止皮表水分蒸发, 由 于呈弱酸性可抑制一些化脓菌及皮肤癣菌的定植; (4)一些暂 驻菌中的机会致病菌作为抗原刺激机体免疫系统, 促进免疫 器官发育, 增强机体免疫功能。相关研究显示对皮肤致病菌 的保护性免疫有依赖于皮肤正常微生物群而非肠道微生物群

(Capone et al., 2011; Gallo1 \& Nakatsuji, 2011)。

\section{Relationship between skin microecology dysbiosis and skin disease}

黄褐斑: 黄褐斑患者皮损区的痤疮丙酸杆菌活菌数明显低 于正常人, 而产色素微球菌、革兰阴性菌的活菌数明显高于 正常人。特应性皮炎: 皮肤常住细菌表皮葡萄球菌在湿疹皮 炎皮损中的定植远低于正常人皮肤, 金黄色葡萄球菌检出率 76 至100\%, 认为与后者相关。银屑病: 患者躯干部皮损变 形菌的比例较健康人皮肤明显增加; 银屑病皮损中链球菌属 高于健康对照; 另外皮损中葡萄球菌和丙酸杆菌含量则显著 低于健康人皮肤。尿布区: 尿布覆盖部位皮肤由于容易出现 尿布疹并继发感染, 白色念珠菌是常见的尿布疹继发感染菌

（Keswick et al., 1987）。金黄色葡萄球菌也经常在患处检 查到, 其他如变形杆菌、肠球菌和绿脓假单胞菌也偶有检测 (Grice, 2014)。

\section{Systematic research and treatment of skin reflects holism of cosmetic science}

皮肤作为机体表面器官, 很容易受到外界环境因素的影 响, 正常情况下通过上述调节机制, 均能够保护好机体健康 和皮肤健康, 免受内外环境损害。但是, 当机体处于亚临床 状态或外界因素达到致病程度时, 上述功能失调, 将导致机
体或皮肤组织、功能学变化, 产生异常的临床表现, 如机体 出现异味, 色泽发生变化, 表面纹理异常等, 甚至出现特征 性皮肤疾病, 头皮屑、痤疮等。这些往往并非局部变化, 或 单因素变化, 是系统性变化。例如, 身体异味, 往往是由于 神经内分泌异常带来的汗腺或皮脂腺腺体分泌异常, 继发微 生态失调, 某些产气菌群活跃等, 使机体产生异常气味。

\section{Rough and dry skin}

皮肤干燥发生在所有年龄, 不分男女、不分区域, 在60岁 以上的人群中，几乎每人都存在着皮肤干燥问题。皮肤屏障 功能主要依赖于皮肤上层的表皮, 也被称为角质层。肩负着 非常重要的体温、气体交换, 防御病原体入侵, 角质层维持 适当的水合作用。在出生时, 婴儿皮肤的水处理性能是独特 的, 不同于成年人。虽然在生命的第一个月相当干燥, 在接 下来的两年中, 皮肤的水含量显着增加, 使最初粗粘的角质 层变得更滑润。但在这个过程中, 皮脂的生产水平, 天然保 湿因子（NMF）包括吸湿混合氨基酸，尿素和其他化合物水 平较低。

在成人, 虽然皮脂分泌不足可能导致皮肤干燥, 但角质层 更复杂的功能障碍, 是引起皮肤干燥的主要原因。通过对年 轻和老年人干燥皮肤研究, 发现干燥皮肤屏障的完整性和相 关的屏障修复能力明显异常。然而, 神经酰胺, 胆固醇, 游 离脂肪酸的分布相似, 功能性皮肤变化是普遍归因于缺乏关 键角质层脂质, 随着年龄的增加角质层间隙中的层状双分子 减少, 脂肪酸酯化神经酰胺比例发生改变, 从而导致皮肤水 分流失的敏感性增加, 特别是在干旱的月份。相对年轻人来 讲, 老年表皮增殖能力下降, 皮肤干燥。

通过对干燥皮肤进行角蛋白分析, 发现不同的角蛋白表达 发生显著变化, 角蛋白 K1和 K10明显减少, 基底角蛋白 K5 和 K14明显增加。干燥皮肤, 外皮蛋白过早表达, 蛋白质组分 成为与角质细胞的角质层 (细胞型) 细胞膜发生交联, 从而 增加细胞刚度。到目前为止, 有关角蛋白的这些研究成果, 被认为是促进、保持和削弱角质层屏障的关键物质。如果这 些生理性物质变化将改变皮肤的水结合能力。由于角质层的 水枯竭, 失去柔韧性的同时, 小裂纹的形成（Liu, 2003）。 皮肤表面晦暗、粗楉、和鳞片状的外观, 较细的糠状鳞片可 以呈现为皮屑。更严重时, 浅表裂缝纵横交错的模式可能被 看到, 看上去就像一个 “干涸的河床上”。有时出现渗出、 结痂, 由于搔抓而出现划痕。干燥的皮肤可能经常伴随着癌 痒和刺痛, 这两者都可能是由皮肤神经纤维直接刺激引起 的。机械的抓挠、摩擦, 或通过刺激性或致敏物质, 可以加 剧释放组胺和其他炎症细胞因子继发, 从而加剧障碍损害。 轻度干燥可以合理保湿在短时间内改善和维护, 更严重的情 
况下, 如特应性皮炎, 可能需要长时间的治疗, 外用皮质类 固醇。然而, 在任何一种情况下, 一般经常使用的闭塞剂、 保湿剂和润肤剂, 和/或再生剂, 利于干燥恢复和减少复 发。

\section{1)Type of moisturizing agent}

角质层是一个互动的、动态的结构, 保持水化可以影响其 屏障功能。保湿霜作为一个复合物, 应被设计为增加角质层 水化或恢复角质层水合的作用。所有的保湿霜将有助于提供 某种形式的临时屏障, 又将起到持续地修复受损的角质层, 保湿剂可分为几大类：保湿剂、润肤剂、封闭剂, 天然保湿 因子/必不可少的蛋白质成分。(1)保湿剂, 是一类涂在皮肤 上时, 能够吸收水分。从理论上, 这些物质可以提高角质层 的水化。典型的保湿剂有甘油、山梨糖醇、尿素、 $\alpha$-醇酸、 糖。(2)封闭剂, 封闭剂可以物理性地阻止水分的从角质层丢 失, 有助于保持水分, 如羊毛脂、矿物油, 硅油, 氧化锌。 这些封闭剂还具有润肤的功效, 进一步提高其改善肌肤整体 性质的能力。(3)润肤剂, 经常与保湿剂一起应用, 渗入角质 细胞之间填充空隙, 达到保湿光滑皮肤的作用, 而不是通常 的闭塞作用。常用润肤剂包括角鲨烯、胆固醇、和脂肪酸。 (4)天然保湿因子, 含有多种天然氨基酸、尿刊酸、无机盐、 糖、乳酸、尿素。许多这些物质具有高效的吸附和束缚水, 让即使在低湿度环境的角质细胞充分水化。大部分的NMF是 来自分解蛋白, 大量富含组氨酸蛋白, 参与新形成的细胞角 蛋白调节。(5)非甾体抗炎化合物, 一类新的化合物, 非甾体 抗炎霜专为干燥, 过敏性皮肤、生物活性脂肪缺乏患者的特 应性皮炎。这些物质在有助于润肤的功效同时, 具有抗炎作 用, 具有低效价的类固醇功效而又不含任何甾体或钙调磷酸 酶抑制剂的性能。这使得皮肤屏障功能得到修复和改善, 增 加皮肤水含量。

\section{Skin pigmentation}

色素沉着或色斑形成机制比较明确, 主要取决于皮肤中黑 素含量, 与四个方面相关：（1）酪氨酸酶与黑素合成;

（2）黑素合成调节: 黑素合成并非黑素细胞自身的孤立事 件, 与内外环境有着密切的联系。(1)内源性调节, 主要为 内分泌因素, 如黑素细胞刺激素(MSH)、促肾上腺皮质激素 (ACTH) 有刺激黑素细胞分泌黑素作用。(2)外源性调节: 食物中酪氨酸、苯丙氨酸含量高时, 黑素产生量就多, 肤色 较黑。（3）信号传导调控：外界的刺激信号因子通过黑素 细胞膜表面的相关受体介导, 经下游信号转导来调控相应的 靶点, 逐级引起细胞质中蛋白质变化, 从而对黑素细胞增殖 分化及黑素生成进行调控。报道较多的传导途径, DAG(甘油
二酯)/PKC(蛋白激酶C)、NO/CGMP/PKG、CAMP/PKA、MAPK 级联途径(丝裂原激活的蛋白激酶)途径。（4）黑素代谢途 径: 黑色素小体的移行途径有两种, 第一种途径是向外移 行, 随角质脱落; 第二种途径, 是向内移行, 经由淋巴系统 从肾脏排出（Brenner \& Hearing, 2008）。

皮肤色素沉着、色斑形成, 主要是黑素细胞受内在和外界 因素的影响, 导致黑素细胞活跃从而合成过多的黑素导致。 体内激素波动, 外部紫外线、慢性炎症、皮肤摩擦损伤是黑 素合成增加导致色素沉着的主要原因。皮肤角质形成细胞 和/或成纤维细胞, 与黑素细胞间存在着旁泌调节连结。它 们产生的细胞因子, 彼此之间相互反应, 对皮肤黑素过度沉 着产生积极的影响。紫外线照射培养的角朊细胞和人正常成 纤维细胞, 其培养液可以诱导培养的黑素细胞DNA合成量增 加, 并存在着剂量一反应关系。角质形成细胞与成纤维细胞 暴露UVA和UVB时所产生的细胞因子是不同的, 角质形成细 胞主要分泌白介素-1（IL-1）、内皮素-1（ET-1）、粒细胞和 巨细胞刺激因子（GM-SCF），而成纤维细胞则分泌干细胞 因子和肝细胞生长因子。在皮肤黑素细胞合成过程中, 角质 形成细胞、成纤维细胞和黑色素细胞之间, 存在着自分泌调 节、旁分泌调节（Cuietal.,2007）。

美白添加剂研究和选择, 并不再是单纯的围绕着黑素合成 环节, 如抑制黑素细胞酪氨酸酶活性, 而是将视野扩展到黑 素细胞的活性调控, 黑素小体排泄或清除的多少等。该种美 白思维不仅达到更好的效果, 还有利于保护肌肤 (Seiberg, 2000）。

\section{Cutis laxa and wrinkle}

衰老的皮肤在表观上体现如下特征: 皱纹、松弛、干燥、 粗燥、色素沉着不均。所以, 研究衰老, 必须建立在研究上 述皮肤粗䊁、干燥、色素沉着机制或解决方案的基础上, 进 一步研究松弛和皱纹的机制和解决方案, 然后综合上述研究 成果, 综合分析，探讨抗衰老方案。

1) The histologic changes of cutis laxa and wrinkle

表皮层: 随着衰老的发生, 皮肤的角质层结构变得不紧 密, 常呈 “网篮状” 图形。角质形成细胞增殖及分化能力, 细胞信号传导功能及修复DNA损伤的能力下降。角蛋白和脂 质代谢紊乱。黑素细胞数量减少, 生命周期缩短, 对生长因 子的反应减弱。郎格汉斯细胞数量减少 $20 \%$ 50\%, 形态异 常, 皮肤免疫功能下降, 皮肤中UVA诱导的免疫抑制活性上 升。真皮表皮连接处：衰老发生过程中，真皮表皮之间的连 接逐渐变弱, 表皮真皮连接处变平、厚度变薄。真皮层: 真 皮层结构的改变是皮肤衰老的主要原因。真皮层中的主要细 
胞为成纤维细胞, 随着衰老的发生, 成纤维细胞数量逐渐减 少，对生长因子的反应减弱，其合成细胞外基质的能力下 降。在衰老的皮肤中总的胶原蛋白含量下降; I型胶原蛋白/ II型胶原蛋白的比例下降; 由于基质金属蛋白酶 (MMPS) 的 增多导致胶原蛋白的降解。葡萄糖胺聚糖是真皮层中存在的 粘多糖, 主要起粘合支持及吸水保湿作用。粘多糖的存在让 皮肤保持丰盈、柔软、水润。皮肤中的葡萄糖胺聚糖包括透 明质酸、硫酸皮肤素、硫酸软骨素。在衰老的皮肤中, 真皮 层透明质酸含量不变, 表皮层透明质酸含量急剧减少。皮下 组织: 因衰老而导致的皮下组织变化情况, 随生理部位的不 同而不同。手背、脸部皮下脂肪随着衰老的发生逐渐减少， 而女性腰部、男性腹部皮下脂肪的堆积随年龄增加而增多

(Farage et al., 2013)。

\section{2) Mechanism of wrinkle formation}

由于皮肤胶原蛋白迅速降解、导致皮肤弹性下降或消失, 从而皮肤出现皱纹。内源性、外源性和机械性因素对有丝分 裂和信号传导的影响, 导致皱纹产生。通过细胞因子或炎性 因子受体，如表皮生长因子（EGF）、白介素-1（IL-1）、肿 瘤坏死因子（TNF- - ）、血小板衍生生长因子 (PDGF) 、血 小板激活因子 (PAF) 受体, 激活酪氨酸激酶和相关调节蛋 白，通过转录因子激活蛋白-1（AP-1）信号传导，激活基质 金属蛋白酶（MMPs）。打破了自然状态下的基质金属蛋白 酶与其抑制剂的平衡, 导致胶原蛋白快速降解。AP-1由两个 亚单位组成, 即C-fos和c-jun。 $\mathrm{c}$-fos的连续表达, 以至于 $\mathrm{c}-\mathrm{jun}$ 在皮肤中表达限制了AP-1活性。有意义的是, 已经明确在年 轻和衰老皮肤中c-fos的表达水平相似, 与年轻皮肤相比, 衰 老皮肤c-jun表达增加。所有这些因子均引起胶原蛋白代谢, 导致前胶原蛋白|型和VII型合成减少、紊乱、结构受到破坏, 基质金属蛋白酶抑制剂（TIMPS）失活，诱导MMPS合成增加

(Slominski et al., 2014; Chen \& Lyga, 2014; Asadamongkol \& Zhang, 2014)。

抗衰老产品应当具有的功效, 抵御有害因素, 滋养和调节 组织细胞, 达到抗衰老目的。配方原则：1）保湿和修复皮 肤的屏障功能；2）促进细胞新陈代谢；3）补充皮肤中的胶 原蛋白和弹性蛋白；4）拮抗内源性和外源性自由基, 抗氧 化；5）防晒；6）修复DNA损伤；7）修复皮肤免疫系统， 提高防御能力；8）减少神经末梢的过度反应, 淡化皱纹

(Zhuang \& Lyga, 2014; Gancevicieneet al.,2012)。

\section{Acne}

青春痘的靶单位是毛囊皮脂腺, 而皮脂腺的发育和分泌直 接受内分泌系统的控制。雄激素作用于皮脂腺导致皮脂溢出
增加是青春痘发生的基础。皮脂腺细胞和毛囊角化细胞的代 谢常需雄激素的刺激, 而毛囊和皮脂腺细胞中存在一些特异 性还原酶, 特别是5a-还原酶。这些酶可使血循环中较弱的雄 激素转化为活性较强的双氢睪酮和睪酮, 后者可引起皮脂腺 的分泌六进。引起角质细胞过度增生, 引起皮脂腺导管上皮 细胞层不断增厚、管径变小、通畅度减弱, 最终导致毛囊皮 脂腺导管急性闭塞, 毛囊隆起而形成粉刺。毛囊过度增殖、 异常皮脂包涵物增加, 导致细胞异常分化或皮脂从毛囊皮脂 腺腔中流出, 该过程中不断出现的各种炎症刺激因子, 导致 临床表现的炎症反应发生, 严重炎症反应往往伴随严重的组 织损害, 形成重度青春痘。随着青春痘的炎症加重, 开放式 青春痘往往伴有致病微生物感染。

青春痘的发病机制基本明了, 简单来说就是: (1)雄激素的 作用: 使皮脂腺活跃, 皮脂分泌增加, 皮肤油淢。(2)毛囊漏 斗部角质细胞粘连性增加, 开口处堵塞。(3)毛囊皮脂腺内的 痤疮丙酸杆菌大量繁殖, 分解皮脂。(4)化学和细胞的介质导 致炎症, 进而化脓, 把毛囊皮脂破坏。痤疮治疗, 主要围绕 中控油、杀菌、抗炎、剥脱表皮等。然而, 临床研究显示, 消费者普遍发现使用产品后出现的皮肤干燥、脱屑、瘙痒症 状，均表现为皮肤屏障遭到损害，尤其是使用不正规的祛痘 化妆品、外用酒精配制的擦剂、使用过热的水洗脸、使用香 㿝、洁面乳、频繁去角质护理, 使患者皮肤屏障遭受损害。 痤疮治疗中导致的屏障损伤, 导致如下后果, 1) 屏障功能 弱, 导致皮肤防御能力降低, 可能导致刺激性物质入侵, 导 致炎症加重；2）屏障功能损伤，导致皮肤发出产生皮脂和 角质的信号, 又进一步加重了皮脂分泌和角化异常；3）屏 障功能损伤导致的显著的干燥、瘁痒、脱屑症状, 加重了患 者对于产品和药品的不耐受性, 使得延误治疗。所以, 在注 意到上述治疗环节后, 还必须加强屏障修护意识 (Tanghetti, 2013)。

\section{The holistic principles of cosmetics formula design}

因此, 对于化妆品配方, 不应堆砌式地添加化妆品功效物 质。化妆品整体观的思想将更全面地指导产品开发, 从而形 成系统的配方体系。

解决皮肤问题, 不能以点代面。保湿产品, 除了补充皮肤 水分和防止水分流失, 更重要的是上调与皮肤干燥相关的蛋 白表达以及加强皮肤的屏障结构, 同时添加一些抗炎活性物 质，降低皮肤对外界刺激的炎症反应。美白产品，传统途径 为抑制黑色素的合成，整体观思想的美白产品开发理念则可 
通过几条途径达到美白，例如抑制黑色素的合成，调节黑素 细胞的活性，促进黑素小体的排泄或清除，添加自由基清除 剂降低色素的沉着。抗衰老产品则可通过保湿, 加固皮肤屏 障，供给皮肤营养，抗氧化，提高皮肤的免疫力以及防晒等 方面来达到抗衰老目的。祛痘类产品可围绕抑菌、抗炎、控 油、加固皮肤屏障，降低皮肤刺激等活性物质的添加，恢复 到较正常的皮肤状态。

上述思想，都体现了化妆品科学的整体观原则，使化妆品 能有的放矢、相得益彰、更全面地改善皮肤状态。将化妆品 产品开发理念与整体观思想有机地结合, 将会取得理想的产 品效果。

\section{References}

Arck PC, Slominski A, Theoharides TC, Peters EM, Paus R. Neuroimmunology of stress: skin takes center stage. Journal of Investigative Dermatology, 126: $1697-1704,2006$.

Asadamongkol B, Zhang JH. The development of hyperbaric oxygen therapy for skin rejuvenation and treatment of photoaging. Medical Gas Research, 4: 1-6, 2014.

Brenner M, Hearing VJ. The protective pole of melanin against UV damage in human skin. Photochemistry and photobiology, 84: 539-549, 2008.

Capone KA, Dowd SE, Stamatas GN, Janeta N. Diversity of the human skin microbiome early in life. Journal of Investigative Dermatology, 131: 2026-2032, 2011.

Chen Y, Lyga J. Brain-skin connection: stress, inflammation and skin aging. Inflammation \& Allergy-Drug Targets, 13: 177-190, 2014.

Cichorek M, Wachulska M, Stasiewicz A, Tymińska A. Skin melanocytes: biology and development. Postepy Dermatologii I Alergologii, 30: 30-41, 2013.

Cui F, She XD, Li XF, Shen YN, Lu GX, Liu WD. Effects of Malassezia isolates on cytokines production associated with melanogenesis by keratinocytes. Acta Academiae Medicinae Sinicae, 29: 196-200, 2007.

Ellerby JH, McKenzie J, McKay S, Gariépy GJ, Kaufert JM. Bioethics for clinicians: 18. aboriginal cultures. Canadian Medical Association Journal, 163: 845-
$850,2000$.

Fang H. Progress of skin microbiome. Chinese Journal of Dermatology, 47: 1-3, 2014.

Farage MA, Miller KW, Elsner P, Maibach H. Characteristics of the aging skin. Advances in Wound Care, 2: 5-10, 2013.

Gallo RL, Nakatsuji T. Microbial symbiosis with the innate immune defense system of the skin. Journal of Investigative Dermatology, 131: 1974-1980, 2011.

Ganceviciene R, Liakou AI, Theodoridis A, Makrantonaki E, Zouboulis CC. Skin antiaging strategies. DermatoEndocrinology, 4: 308-319, 2012.

Gandarillas A. The mysterious human epidermal cell cycle, or an oncogene induced differentiation checkpoint. Cell Cycle, 11: 4507-4516, 2012.

Grice EA. The skin microbiome: potential for novel diagnostic and therapeutic approaches to cutaneous disease. Seminars in Cutaneous Medicine and Surgery, 33: 98-103, 2014.

Guo HW, Hao FJ. Research progression of independent HPA system in skin. Chinese Journal of Dermatovenereology, 23: 841-844, 2009.

Huang XR. Reductionism and its historical significance. Journal of Jiangxi University of finance and Economics, 4: 74-78, 2008.

Jang JW, Gao SZ, LI B, Zhao SM. Research progress of adipocytokines. Journal of Anhui Agricultural Sciences, 40: 12105-12107, 2012.

Keswick BH, Seymour J, Milligan MC. Diaper area skin microflora of normal children and children with atopic dermatitis. Journal of Clinical Microbiology, 25: 216-221, 1987.

Kunitz SJ. Holism and the idea of general susceptibility to disease. International Journal of Epidemiology, 31: 722-729, 2002.

Liang R, Yang C, Tang W, Deng RX. Reflection and exploration on the teaching of sensory evaluation in cosmetics. Guangdong Chemical Industry, 42: 185188, 2015.

Liao X, Liu HW, Cheng B. Effect of cutaneous neuroendocnne system on epidermal stem cells and biological significance. Chinese Journal of Aesthetic Medicine, 21: 2282-2284, 2012.

Liu CH. Alterations of aged skin. Journal of Clinical 
Dermatology, 32: 113-116, 2003.

Liu W, Chen QS, Gong SZ, Wu ZQ. Introduction of human epidermal growth factor and its application in cosmetics. Detergent \& Cosmetics, 37: 36-38, 2014.

Liu XM, Lei TC. A functional equivalent of hypothalamic pituitary adrenal (HPA) axis exists in the skin. International Journal of Dermatology \& Venereology, 35: 257-260, 2009.

Man MQ, Liu L, Lu CZ. Stratum corneum hydration and its impact on cutaneous biology. Journal of Clinical Dermatology, 37: 816-818, 2008.

Mao JR. The systemic whole concept in history. Chinese Journal of Systems Science, 14: 87-90, 2006.

Prohic A, Simic D, Sadikovic TJ, Krupalija-Fazlic M. Distribution of Malassezia species on healthy human skin in Bosnia and Herzegovina: correlation with body part, age and gender. Iranian Journal of Microbiology, 6: 253-262, 2014.

Qi HW, Zhu JL. Ecological philosophy and its ecological aesthetic wisdom in Shengsheng of 〈Zhou Yi〉. Shandong Social Sciences, 5: 34-38, 2013.

Ren JH, Li CH. Natural holism of Sancai theory in the confucian "harmony between man and nature". Theory Journal, 5: 88-91, 2006.

Röhl J. Mechanisms in biomedical ontology. Journal of Biomedical Semantics, 3: 2-14, 2012.

Roosterman D, Goerge T, Schneider SW, Bunnett NW, Steinhoff M. Neuronal control of skin function: the skin as a neuroimmunoendocrine organ. Physiological Reviews, 86: 1309-1379, 2006.

Seiberg M, Paine C, Sharlow E, Andrade Gordon P, Costanzo M, Eisinger M, Shapiro SS. Inhibition of melanosome transfer results in skin lightening. Journal of Investigative Dermatology, 115: 162-167, 2000.

Slominski AT, Kleszczyński K, Semak I, Janjetovic Z, Zmijewski MA, Kim TK, Slominski RM, Reiter RJ, Fischer TW. Local melatoninergic system as the protector of skin integrity. International Journal of Molecular Sciences, 15: 17705-17732, 2014.

Tang DM. From image to abstract: the birth of aristotelian logical theory. Journal of Southwest China Normal University, 28: 23-27, 2002.

Tanghetti EA. The role of inflammation in the pathology of acne. The Journal of Clinical \& Aesthetic Dermatology, 6: 27-35, 2013.

Tang YH. On holism of Laozi'Dao. Journal of Hunan Industry Polytechnic, 12: 45-63, 2012.

Torday JS. A central theory of biology. Medical Hypotheses, 85: 49-57, 2015.

Wen RQ, Lu WH, Yan CX, Zhang HM, He GC. Basic and clinical research of the relationship between the activity of tyrosinase and the synthesis of melanin. Chinese Journal of Aesthetic Medicine, 23: 20282031, 2014.

$\mathrm{Xu}$ J, Wang YJ. Discussing the science of traditional Chinese Medicine by the holism of 〈Huangdi Neijing〉. Acta Chinese Medicine and Pharmacology, 42: 1015, 2014.

Zhuang Y, Lyga J. Inflammaging in skin and other tissues - the roles of complement system and macrophage. Inflammation \& Allergy-Drug Targets, 13: 153-161, 2014. 


\section{국문초록}

\section{화장품과학의 전체론}

동은묘, 추현영, 맹홍*

북경공상대학 화장품과학기술학과, 북경, 중국

목적: 화장품피부과학은 독립적인 학문이면서 생명과학, 화학, 포장설계 등 20 개이상 분야를 포함한 종합적인 학문이다. 화장품피 부과학은 초기 간단한 피부조직에서부터 시작하였으나 피부가 시상 하부- 뇌하수체- 부신간의 내분비조절작용을 한다고 인식됨에 따라 피부는 내분비 신호전달의 대상기관으로 인식되기 시작했다. 피부과학은 피부의 매크로 이해로부터 현재의 피부미생물과 같 은 마이크로 단계의 이해로 발전되었다. 그래서 본 연구는 피부의 전체론을 제시해서 피부문제를 해결하려 한다.

방법: 고금중외 모든 국가의 형성과 전체론의 계기로 화장품과학전체론 및 화장품학과 연관된 학과 특히 피부과학의 발전과정을 설 명하였다.

결과: 본 연구는 피부과학의 발전과정을 총 정리하고 전반적인 관점을 설립하여 각종피부문제를 해결하려 한다.

결론: 화장품과학의 전체론은 화장품과학 발전의 필연적인 추세로 될 것이라고 사료된다.

핵심어: 전체론, 화장품과학, 피부과학, 피부증상, 화장품 포뮬러 


\section{中文摘要}

\section{化妆品科学的整体观}

董银卯, 邱显荣, 孟宏*

中国北京工商大学化妆品科学与技术科, 北京, 中国

目的: 化妆品科学作为一门独立的学科, 它是集生命科学、化学、包装设计等20个以上学科的一门综合性学科。通过对皮肤组织 的简单认识了解, 以及对皮肤组织下丘脑-垂体-肾上腺系统的深入了解, 皮肤是下丘脑-垂体-肾上腺的内分泌靶器官, 即多种内 分泌信号作用的靶器官。从皮肤科学在宏观角度上了解皮肤, 到目前以微生物群落等微观角度理解皮肤, 提出了整体观来解决 皮肤问题。

方法: 受古今中外所有国家的形成和整体论的意义的启发, 我们阐述了化妆品科学整体观及化妆品相关学科, 特别是皮肤科学 的发展历程。

结果: 从总结皮肤科学的发展过程中, 建立了一个整体观来解决各种皮肤问题。

结论: 化妆品科学的整体观将成为化妆品科学发展的必然趋势。

关键词: 整体观, 化妆品科学, 皮肤科学, 皮肤症状, 化妆品配方 